\title{
Species collage
}

\section{A new study reports the first viable rat- mouse chimeras and uses rat induced pluripotent stem cells to rescue organ deficiency in mice.}

Far from the fire-breathing female monster of Greek mythology, 'chimeras', for biologists, are important methodological tools. In biological research, when embryonic cells from two different organisms are mixed at the blastocyst stage and allowed to generate adult animals, the resulting hybrids, composed of cells from both donors, are called chimeras.

Intraspecies chimeras are created by transplanting embryonic cells from an animal with one trait into an embryo of an animal with a different trait, both belonging to the same species. The production of mouse chimeras from different mouse strains has been very important in our understanding of how embryos form and cell lineages contribute to them. Alternatively, when cells from two different species are mixed, interspecies chimeras can be generated. Until now, however, the only viable intergeneric chimera resulted from the combination of embryos from a goat and a sheep, and prior attempts to generate viable mouse-rat chimeras had been unsuccessful.

Hiro Nakauchi at the University of Tokyo and his colleagues decided to revisit this challenge using modern technologies. Instead of injecting cells of pre-blastocyst origin from one species into the blastocyst of another species, they used embryonic stem cells (ESCs) and induced pluripotent stem cells (iPSCs) as donor cells. The group injected fluorescently labeled mouse or rat iPSCs (or ESCs) into rat or mouse blastocysts, respectively, and implanted these blastocysts into foster mothers of the same origin as the blastocyst. The resulting embryos were born and gave rise to viable adult rat-mouse chimeras.

Surprisingly, these chimeras were not 50:50 rat-mouse hybrids. The size of the animals, for one, was entirely determined by the species of the host blastocyst. "We didn't expect the size of these chimeras to be so different," explains Nakauchi, "and what determines this is at this moment still a mystery."

But Nakauchi and colleagues did not stop there. With a background in immunology and hematopoietic stem cells, Nakauchi was very interested in determining whether donor cells of one species could rescue a defect in organ development in a recipient of

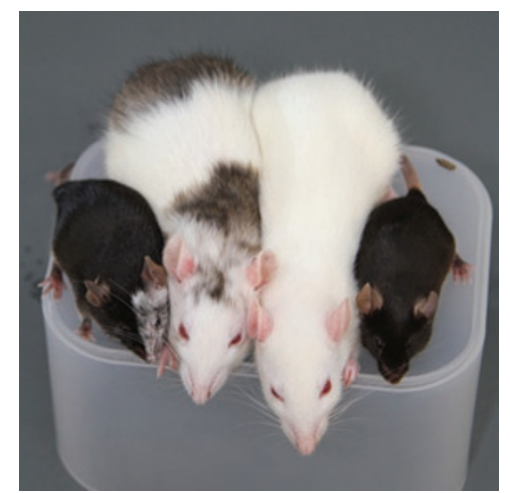

Mouse-rat chimeras. From left to right, chimera resulting from rat iPSCs injected into a mouse blastocyst, chimera resulting from mouse iPSCs injected into a rat blastocyst, wild-type rat and wild-type mouse. Image courtesy of Hiro Nakauchi.

a different species.

To do this, the group used a technique called 'blastocyst complementation', which is used in immunology to rescue defects in specific cell lineages by injecting mouse ESCs that contribute to the missing cells in the developing embryo. Nakauchi reasoned that the same basic principle could apply to compensate the defect of a missing organ, and he and his colleagues injected rat iPSCs into recipient mouse blastocysts lacking a gene essential for pancreas development. The resulting chimeras were viable and had a functional pancreas entirely derived from the donor rat cells.

However, the survival rate of these chimeric animals was low, and the transplanted cells contributed to many tissues other than the pancreas. To address this, Nakauchi's group intends to use stem cells committed to specific lineages and transplant them at later stages of development.

Ultimately, Nakauchi wants to use this technology to make human organs in pigs-a goal with numerous biological and technical challenges, not to mention the many ethical and political barriers. In the meantime, the group is busy generating new combinations of rat-mouse chimeras and rescuing rodent deficiencies in the thymus, kidney, liver and heart.

\section{Erika Pastrana}

\section{RESEARCH PAPERS}

Kobayashi, T. et al. Generation of rat pancreas in mouse by interspecific blastocyst injection of pluripotent stem cells. Cell 142, 787-799 (2010). 